\title{
Post-traumatic Frontal Lobe Epilepsy with Structural Changes: Excellent Results After Cortical Resection
}

\author{
Arthur Cukiert, André Olivier and Frederick Andermann
}

\begin{abstract}
Background: The observation that resection of structural lesions of the frontal lobe provides an effective treatment of intractable epilepsy has been made by surgeons specializing in this field for over sixty years! However, the excellent results achieved when such resections are coupled with the use of modern diagnostic and operative technologies are not fully appreciated by the modern clinician. Methods: We review the results of resection of large post traumatic frontal lesions in six patients with intractable frontal seizures. No invasive EEG recording was required pre-operatively. Results: The imaging characteristics of the underlying lesions, usually due to depressed skull fractures are illustrated. Surgical treatment rendered five patients seizure free and the sixth had over $85 \%$ improvement in seizure frequency. Conclusions: Modern imaging permits ready visualization of the post traumatic lesions with cause intractable seizures. It is important to recognize that resection of these post traumatic scars usually leads to excellent seizure control.
\end{abstract}

RÉSUMÉ: Épilepsie frontale post-traumatique accompagnée de changements structuraux: excellents résultats de la résection corticale. Introduction: Les chirurgiens se spécialisant dans la chirurgie de l'épilepsie ont constaté depuis plus de soixante ans (Foerster and Penfield, 1930) que la résection de lésions structurales du lobe frontal constitue un traitement efficace de l'épilepsie résistante au traitement. Cependant, les excellents résultats obtenus quand ce type de résection est associé à l'utilisation de technologies diagnostiques et opératoires modernes ne sont pas suffisamment reconnus par le clinicien moderne. Méthodes: Nous avons revisé les résultats de résections de lésions frontales post-traumatiques étendues chez six patients qui présentaient des crises d'épilepsie frontale rebelles. Aucun enregistrement EEG invasif préopératoire n'a été nécessaire. Résultats: Nous présentons par l'imagerie les caractéristiques des lésions sous-jacentes, généralement dues à des fractures du crâne avec dépression. Cinq patients n'ont plus présenté de crises après la chirurgie, alors que le sixième a eu une réduction de la fréquence des crises de $85 \%$. Conclusions: L'imagerie moderne permet une visualisation facile des lésions post-traumatiques qui causent les crises d'épilepsie rebelle. Il est important de savoir que la résection de ces lésions post-traumatiques entraîne généralement un excellent contrôle des crises.

Can. J. Neurol. Sci. 1996; 23: 114-117

"The important feature is that the removal is radical, clean, and directed toward the lesion responsible for the attacks".

\section{Foerster and Penfield $1930^{\prime}$}

After coming to Montreal in 1928, Wilder Penfield spent six months with Otfried Foerster in Breslau acquiring experience in the surgical treatment of epilepsy. Together they recognized the importance of resecting the epileptogenic lesion and reported complete cessation of seizures in five of seven patients: one was improved and one had insufficient follow-up. These results were quite remarkable considering that they were obtained sixty years ago.'

In contrast, the results of frontal lobe resections for treatment of epilepsy have since been disappointing. This was particularly so when no structural lesion in the frontal lobe could be identified and removed. ${ }^{2,3}$

Frontal lobe seizures usually have a widespread cortical generator when compared to temporal lobe seizures. It is difficult to decide even with invasive recordings whether the seizure pattern is specific to certain areas of origin within the frontal lobe or whether it is due to spread of the epileptic discharge. Despite this, attempts have been made to ascribe different patterns of seizures to specific areas within the frontal lobe. In addition to clinical and EEG features increasing attention has been directed to assessment of frontal structures by modern imaging techniques. ${ }^{4,5}$ It has become apparent that certain patients do much better than others following resection of frontal epileptogenic tissue. Recent experience suggests that frontal lobe epilepsy should be considered according to the etiology and imaging findings in addition to the clinical and electrographic features. ${ }^{6-9}$

In this paper, we describe a specific group of patients with post-traumatic frontal lobe epilepsy associated with structural lesions which can be demonstrated with current imaging studies.

From the Department of Neurology and Neurosurgery, McGill University and the Montreal Neurological Institute and Hospital, Montreal. RECEIVED JULY 18, 1995. ACCEPTED IN FINAL FORM DECEMBER 20, 1995. Correspondence to: Dr. Frederick Andermann, Montreal Neurological Institute and Hospital, 380I University Street, Montreal, Quebec, Canada H3A 2B4 
Resection of these areas of visible damage obliterated the seizures in five of the six patients and improved those of the remaining one.

\section{Patient Material}

Six patients with intractable frontal lobe epilepsy related to direct impact head trauma were studied (Table). They were selected because of their similar histories and imaging findings, from a consecutive series of 28 patients with frontal lobe epilepsy operated by one of us (A.O.). They were the only patients in this series with such histories and imaging finding. Mean age at the time of injury was 11.5 years (5-21) and at surgery 24 years (18-42 years). Mean latency to the development of seizures after trauma was 5.4 years $(0.8-15$ years). Five patients first presented with a depressed and one with a linear skull fracture sustained at the time of an accident. Clinical seizure patterns included complex partial $(n=5)$, tonic-clonic $(n=5)$ and partial motor $(n=1)$ attacks. Mean monthly seizure frequency was 13 (4-60 per month). In all patients neuroradiological studies showed focal atrophy. In 5, frontal wedge-shaped gliotic lesions were present. Contralateral lesions were not apparent on CT or MRI. Extensive surface EEG investigation was performed. Focal epileptogenic discharges were found in all patients, at times associated with generalized spike and wave. EEG abnormalities were restricted to one frontal lobe in 4 patients, bifrontal in one and more widespread in another. This patient had multifocal EEG abnormalities. Invasive preoperative recordings were not carried out.

Electrocorticography (Ecog) was carried out prior to resection in 5 patients, and following resection in 3 . Five had a right frontal resection and one a left frontal removal. Surgery consisted of removal of the visible destructive scar and of surrounding gliotic tissue with additional Ecog guidance.

Mean follow-up was 2.6 years ( $1-5$ years). Five patients were seizure-free. In the one patient with widespread multifocal epileptic abnormalities, resection of the lesion led to an $85 \%$ reduction in seizure frequency. All were still taking antiepileptic medication, usually monotherapy with lower doses and levels compared to the preoperative regimen.

No major neurologic symptoms or signs and, in particular, no increase in frontal lobe deficits occurred as a result of surgery.

\section{Illustrative Patients}

\section{Patient $I$}

A 42-year-old man sustained a compound depressed skull fracture and brainstem injury at the age of 10 years which left him comatose for two months. Fifteen years later he developed complex partial seizures with automatisms occurring 4 times a month. General physical examination was unremarkable. Ictal and interictal recordings showed epileptic activity over the right infero-lateral and mid-frontal regions with subsequent spread to the right infero-mesial and mid-temporal areas. An MRI scan disclosed a right frontal wedge-shaped area of frontal atrophy surrounded by gliosis and ipsilateral "ex vacuo" dilatation of tip of the frontal horn (Figure 1A).

Under general anaesthesia, a right frontal craniotomy was performed. Electrocorticography showed active spiking over the first, second and third frontal gyri. Maximal activity was noted rostrally to the brain scar. Surgery consisted of the removal of the cicatrix and all gliotic tissue located rostrally to this lesion. A post-operative MRI documented the extent of the removal (Figure 1B). He is seizure free after 2.5 years of follow-up.

\section{Patient 5}

This 19-year-old female was the victim of a severe ski-doo accident resulting in a depressed skull fracture with exposure of brain at the age of 9 years. She was treated surgically and recovered well. Two years later, complex partial seizures with automatisms and twitching of the left face started and occurred at a frequency of 6 per month. Since then, she also had sporadic generalized tonic-clonic seizures. General physical examination was normal. A computerized tomographic (CT) brain scan disclosed a wedge-shaped area of hypodensity in the anterior right frontal region with atrophic dilatation of the ipsilateral frontal horn (Figure 1C). Ictal and interictal EEG recordings showed epileptic discharges arising from the right frontocentral convexity.

Under general anesthesia, a right fronto-temporal bone flap was elevated. Electrocorticography showed maximal epileptic activity behind the frontal scar over the second and third frontal gyri. One electrographic seizure was recorded from that area. No epileptic activity was recorded from the temporal lobe. Surgery consisted in the removal of the meningo-cerebral cicatrix including surrounding gliotic tissue. She was seizure-free after 4 years of follow-up. A post-operative scan documented the extent of the resection (Figure ID).

\section{Patient 6}

An 18-year-old man fell from a horse at the age of 5 . Nine years later, he developed complex partial seizures with automatisms and secondarily generalized tonic-clonic attacks. General physical examination was unremarkable. He was of good intelligence but exhibited lack of initiative and drive. EEG recordings showed synchronous spike and wave epileptic abnormalities over both frontal lobes with a clear predominance over the right frontal lobe where independent spiking was also seen. An MRI scan showed a wedge-shaped right frontal lesion surrounded by gliosis (Figure IE).

Under general anaesthesia, a right frontal craniotomy was performed. Electrocorticography disclosed epileptic activity over the cortex just caudal and posterior to the mesially located scar. Surgery consisted in the removal of the gliotic tissue in the first and second frontal gyri and over the mesial surface. A post-operative MRI documented the extent of resection (Figure 1F). The patient is seizure free after a oneyear followup.

A diagram of these three patients' lesions and resections is shown in Figure 2

Table: Clinical features and outcome of surgical treatment in six patients with frontal epilepsy following trauma.

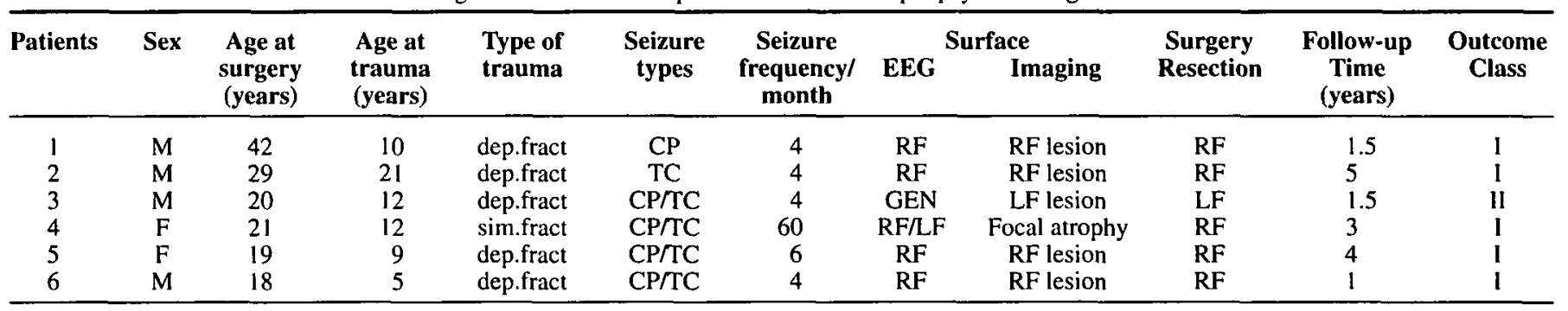

$\mathrm{M}=$ Male $; \mathrm{F}=$ Female $;$ dep = depressed $;$ sim = simple $; \mathrm{CP}=$ complex partial; $\mathrm{TC}=$ tonic clonic $; \mathrm{RF}=$ right frontal; $\mathrm{LF}=$ left frontal; Outcome class = Olivier's classification; I = seizure free; II = pt $3,85 \%$ improvement in number of attacks. 

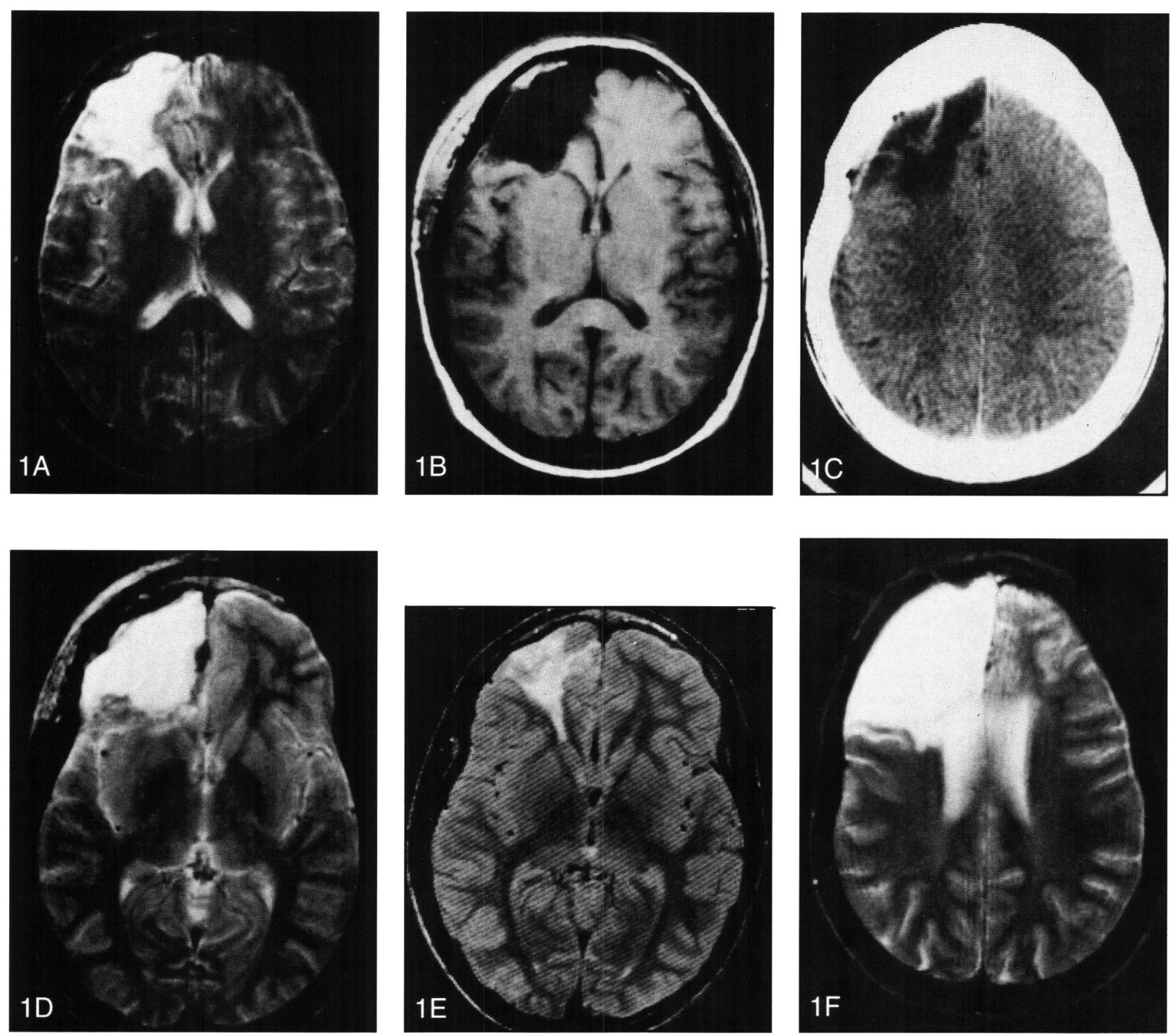

Figure 1: Pre- and post-operative magnetic resonance or CT scans in Patients I, 5 and 6. A and B: Patient $I ; C$ and D: Patient 5; E and F: Patient 6. Post-operative scans show the most cranial level of the resection.

\section{Discussion}

The identification of epileptogenic areas involving the frontal lobe has always been more difficult compared to those located in temporal areas. The results of epilepsy surgery vary proportionally to our ability to remove a specific epileptogenic area. ${ }^{9,10}$

The poor results obtained from frontal cortical removals ${ }^{11,12}$ are likely related to the fact that well localized frontal foci ${ }^{13}$ associated with refractory epilepsy are not documented frequently. Patients with frontal lobe epilepsy caused by head trauma in which the epileptogenic area is the result of a localized direct impact seem to be an exception to this general trend. These lesions are often due to a remote depressed skull fracture and show a well-defined meningo-cerebral cicatrix. The good results obtained after cortical resection in these patients are probably related to the nature of the brain lesion underlying their epileptic syndrome. Direct impact head trauma associated with a depressed skull fracture without significant acceleration/deceleration injuries seems to generate a focal lesion.' Including the lesion demonstrated by imaging in the resection is probably an important determinant of the favourable results. The emphasis on imaging findings does not negate the importance of EEG investigation. There was wide variation in the time of onset on seizures after the trauma but this did not correlate with the result of surgery. A distinctly worse outcome is usually seen after frontal lobe resections carried out in patients with no visible lesion in the MRI. ${ }^{14}$

All patients became seizure free with the exception of the one who had multifocal epileptic discharges indicative of more 


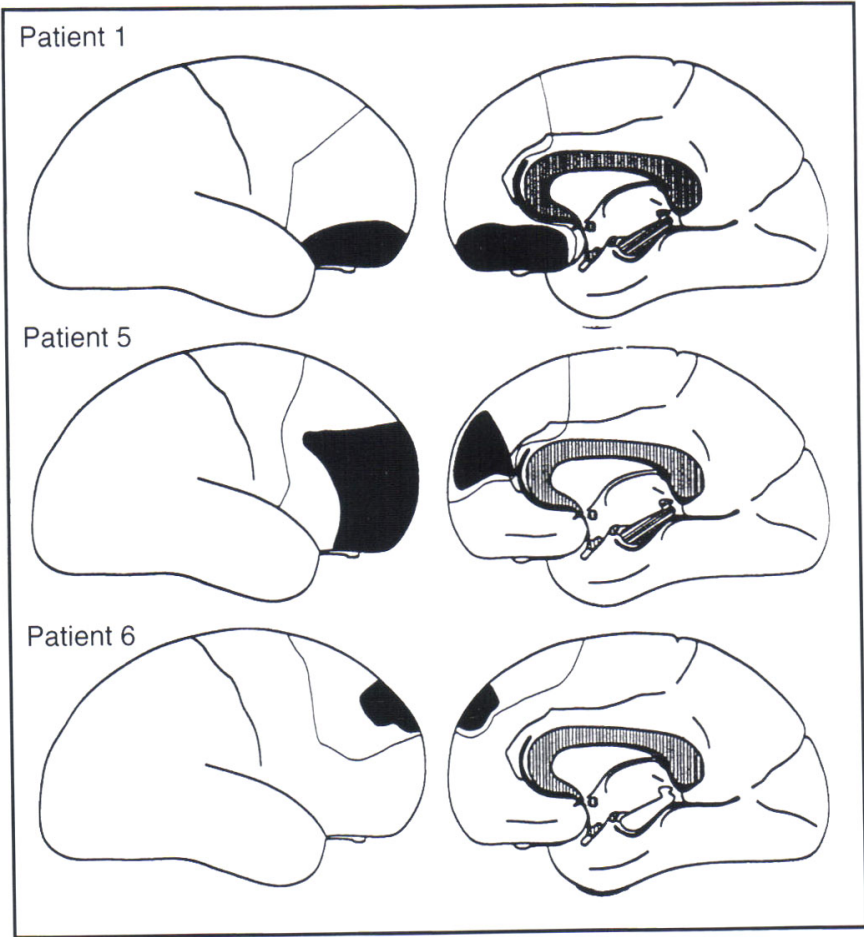

Figure 2: Diagram of the topography of the lesions (black area) and cortical resections (areas anterior to the lines) in Patients $I, 5$ and 6 (A, $B$, and $C$ respectively) as seen in a planar map of the convexity (left) and mesial surface (right).

widespread abnormality. Even in this patient the results of surgery were worthwhile. This type of multifocal disturbance must be distinguished from patients who, in addition to focal epileptic abnormalities, had bilateral spike and wave discharge. Bilateral spike and wave has been described to be related to mesial frontal involvement by Tükel and Jasper ${ }^{15}$ who also recognized that it may disappear after resection. It does not represent a contra-indication to resective surgery in the presence of a unilateral lesion.

The patients did not develop new or additional clinical evidence of frontal lobe dysfunction. This possibility is always a source of concern when surgery in the frontal lobe is considered. The disability caused by the seizures is usually considered significantly greater than potential neuropsychological changes due to surgery. In the presence of a destructive lesion of this type increased frontal lobe symptoms do not seem to occur after surgical resection. Documentation by neuropsychological testing will be reported separately.

The strategy of frontal lobe resections for intractable epilepsy remains an important challenge since the patients are usually severely disabled by their seizures. The patients described here represent a privileged group responding very well to cortical resection. They have been much easier to identify with modern imaging techniques. These individuals resemble in many respects those with focal cerebral cicatricial lesions due to gunshot wounds and perinatal vascular occlusions operated by Foerster and discussed by Penfield. ' Patients with post-traumatic lesions should be recognized among those with intractable frontal epilepsy who, as a group, respond much less well to surgical resection.

\section{REFERENCES}

1. Foerster $O$, Penfield $W$. The structural basis of traumatic epilepsy and results of radical operation. Brain 1930; 53: 99-119.

2. Quesney LF, Olivier A, Andermann F, Gloor P. Pre-operative EEG investigation in patients with frontal lobe epilepsy; trends, results and pathophysiological considerations. J Clin Neurophysiol 1987; 4: 208-209.

3. Williamson PD, Spencer DD, Spencer SS, Novelly RA, Mattson RH. Complex partial seizures of frontal lobe origin. Ann Neurol 1985; 18: 497-504.

4. Waterman K, Purves SJ, Kosaka B, Strauss E, Wada J. An epileptic syndrome caused by mesial frontal lobe seizure foci. Neurology 1987; 37: 577-582.

5. Quesney LF, Constain M, Fish DR, Rasmussen T, The clinical differentiation of seizures arising in the parasagittal and antero-lateral frontal convexities. Arch Neurol 1990; 47: 677-679.

6. Williamson PD, Spencer SS. Clinical and EEG features of complex partial seizures of extra-temporal origin. Epilepsia 1986; 27 : S46-S63.

7. Pedley TA, Tharp BR, Herman K. Clinical and electro-encephalographic characteristics of midline parasagittal foci. Ann Neurol 1981; 9: 142-149.

8. Polkey CE. Cortical resections outside the temporal lobe for intractable epilepsy - excluding multilobar resections and hemispherectomy. Acta Neurochir 1990; 50 (Suppl.): 128-130.

9. Rasmussen T. Characteristics of a pure culture of frontal lobe epilepsy. Epilepsia 1983; 24: 482-493.

10. Adler J, Erba G, Winston KR, Welch K, Lombroso CT. Results of surgery for extratemporal partial epilepsy that began in childhood. Arch Neurol 1991; 48: 133-140.

11. Talairach J, Bancaud J, Szikla G, et al. Approche nouvelle de la neurochirurgie de l'epilepsie (methodologie stereotaxique et resultats therapeutiques. Neurochirurgie 1974; 20 (Suppl. 1): 240.

12. Talairach J, Bancaud J, Bonis A, et al. Surgical therapy for frontal lobe epilepsies. In: Chauvel P, Delgado-Escueta A, eds. Advances in Neurology v. 57. New York: Raven Press, 1992: 707-732.

13. Tharp BR. Orbito-frontal seizures. An unique electroencephalographic and clinical syndrome. Epilepsia 1972; 13:627-642.

14. Cascino GD, Kelly PJ, Hirschom KA, Marsh WR, Sharbrough FW. Stereotactic resection of intra-axial cerebral lesions in partial epilepsy. Mayo-Clin-Proc 1990; 65: 1053-1060.

15. Tükel $\mathrm{K}$, Jasper $\mathrm{H}$. The electroencephalogram in parasagittal lesions. Electroencephalogr Clin Neurophysiol 1952: 4: 481-494. 